\title{
Hippocampal Spike-Slow Wave Phase Relations after Midbrain Transection in Rabbits
}

\author{
PETEK CUYLE ${ }^{1}$ \\ Department of Anatomy, The University of Michigan, Ann Arbor, Michigan 48109
}

Received June 14, 1976; revision received July 28, 1976

\begin{abstract}
The purpose of this investigation was to examine the phase relations of action potentials of single cells in CA1 to the rhythmical slow waves induced by eserine in the rabbit. All animals had an intercollicular decerebration so only structures rostral to the cut contributed to the results obtained. Spikeslow wave phase patterns were compared with a random model using the $\chi^{3}$ test. Nonrandom patterns $(P<0.01)$ characterized 22 of 47 cells tested. With use of digital filters, consistent phase patterns were observed even if the slow wave duration varied by as much as $100 \mathrm{msec}$. Comparing unit firings with nonconcurrent slow wave cycles showed the patterns were phase related, not occurring by chance or coincidence. Apparently clusters of CA1 pyramidal cells fire phase-related spikes.
\end{abstract}

\section{INTRODUCTION}

From previous findings $(10,16,17,19)$ one might surmise that firing patterns of cells in the hippocampus that correlate with rhythmical slow waves were related by a common coupling mechanism. Chance or coincidence could relate the events even if no coupling were present. Periodic or harmonic signals related by chance would have seemingly apparent relationships. Earlier investigations indicated slow wave correlations over extensive regions (4) of the cornu Ammonis CA1 (23) and firings of CA1 theta cells (26), but not CA3 neurons (25), werc consistent during certain movements and stages of rapid eye movement sleep. Because these and other behavioral $(18,30,31,33,35)$, evoked-response $(15,20)$, and drug (29) studies suggested strong signal correlations (11) in a variety

1 The author expresses appreciation to Mr. W. L. Brudon for assistance with the figures and to Ms. Camilla Broman for typing the manuscript. Project support was from a University of Michigan H. H. Rackham Research Grant and Fellowship and from the University of Michigan Medical School General Research Support Grant RR 05383. 
of species (32), spike ${ }^{2}$-phase relations of single CA1 cells to the rhythmical slow waves were investigated. Whereas a midbrain decerebration does not abolish the arecoline-evoked regular waves (22) but could possibly disrupt correlated firings, this preparation was used in an attempt to dissociate the two signal types which may be generated by different kinds of cells.

\section{METHODS AND MATERIALS}

Fourteen rabbits, each weighing between 2 and $3.5 \mathrm{~kg}$ and of either sex, were anesthetized with ether or pentobarbital sodium $(30 \mathrm{mg} / \mathrm{kg}$ body weight) and ether. Stainless-steel microelectrodes (14) coated with Insl-X were coupled to high-input impedance probes. Local slow waves were filtered $(0.3$ to $70 \mathrm{~Hz})$ from the microelectrodes. Amplifier bandwidth for unit potentials was flat from 100 to about $4000 \mathrm{~Hz}$. Concentric bipolar recording electrodes with intertip distances of nearly $0.5 \mathrm{~mm}$ were stereotaxically placed at dorsal (area dentata, P 5.0, L 5.0, and V +5.0) and ventral [CA3, P 5.0, L 8.0, and V -3.0, atlas (5)] positions in the hippocampal arch. Differential amplifier low and high half-amplitude cutoff frequencies were 0.3 and $70 \mathrm{~Hz}$, respectively. Physostigmine salicylate ( 0.25 to $0.35 \mathrm{mg} / \mathrm{kg}$ body weight) dissolved in physiologic saline was administered via a marginal ear vein at least $1.5 \mathrm{hr}$ after discontinuation of the ether anesthesia when the intercollicular midbrain transection was performed. Slow waves and unit potentials were stored on magnetic tape (amplifier response flat from about 0 to $3000 \mathrm{~Hz}$ ). Unit recording sites were marked (14). Unit data were excluded in all cases where metal deposits were not found or were located in nonhippocampal structures. The levels and completeness of the midbrain transections were histologically controlled.

Details of slow wave and action potential sampling procedures are given elsewhere (6). Briefly, a pulse was generated when the unit potential or slow wave crossed a voltage level. Because one cycle of slow wave activity crosses the level at its beginning and end, the crossing times can be used to measure the slow wave duration. Detection of pulses applied to Prime Computer sense lines resulted in storage of real-time clock values used to measure slow wave cycle periods and to compute spike-phase values. Measurement resolution was about $\pm 0.25 \mathrm{msec}$. The duration of each slow wave was displayed in a period or cycle histogram (bindwidth 20 msec) whereas each spike-phase value was plotted in a phase histogram (phasegram, bindwidth 18 or $36^{\circ}$ ). The probability of spike occurrence

2 The term spike or unit potential is used in this paper to indicate the occurrence of a single action potential and does not suggest a potential is a complex spike. Although a complex spike (22) has been considered as one action potential having different components, each unitary component would be evaluated as a point process in this signal analysis. 


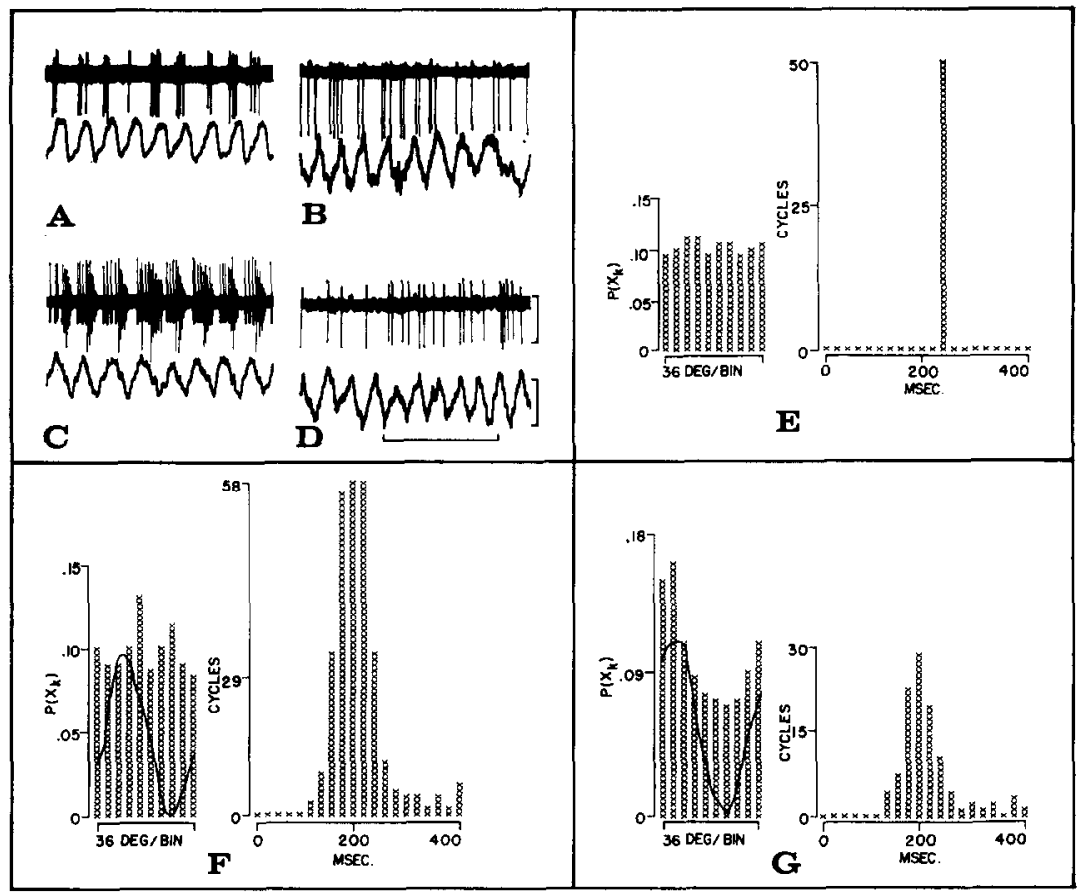

FIG. 1. CA1 spike data, phasegrams, and cycle histograms. Spikes in A have more ordered relations to local slow waves than those in $B$ and D. Spikes in C are from multiple cells. Random model data (E) were obtained from two synthetic nonharmonic signals. Phasegrams $F$ and $G$ (leftmost displays with superimposed averaged slow waves) taken from trains $D$ and $B$, respectively, indicate random patterns $(P>0.05)$. Each $\mathrm{X}$ on the cycle histograms (F, G, rightmost displays) indicates the duration of a slow wave utilized for spike measurement (s) given in the accompanying phasegram. Note duration variations of slow waves. Voltage scales: spikes, $300 \mu \mathrm{V}$, EEG, $500 \mu \mathrm{V}$. Time scale, $1 \mathrm{sec}$.

$P\left(X_{k}\right)$ in a given bin, $k$, is given by $P\left(X_{k}\right)=n\left(X_{k}\right) / N$ where $n\left(X_{k}\right)$ is the number of spikes in the $k$ th bin, and $N$ is the total for all bins. More formalized mathematical treatment of the measurements, the digital filtering procedure, data sequence shuffling, and other considerations are given elsewhere (7). All digital computer programs were written in assembly level language. Averaged slow waves (400 points/sec) computed with a Nuclear Chicago data retreival computer were plotted by a recorder then traced by hand onto phasegrams.

\section{RESULTS}

Phasegram Distributions. In a random spike-slow wave phase model, spike occurrence and nonoccurrence times are not biased. The phasegram (Fig. 1E) has a nearly rectangular-shaped distribution. All neuronal data 
were assumed to represent a random phase pattern unless quantitatively demonstrated otherwise. The $\chi^{2}$ test (3) was used to compare neuronal spike-slow wave phase distributions to the random model. Contingency tables were constructed of phasegram bin values, $\chi^{2}$ values were computed, and the number of degrees of freedom was determined. Standard confidence levels for rejecting the null hypothesis were used for classifying the phasegrams.

Phasegrams of 22 cells differed significantly $(P<0.01)$ from the random model whereas phasegrams of 25 cells did not $(P>0.05$, Fig. $1 \mathrm{~F}$ and $G$ ). Phasegram bin probabilities for 47 cells were grouped and are displayed in Fig. 2. The graph indicates percentages of different bin probability values obtained from the distributions.

The unit discharges were clearly graded in that not all phase patterns were alike. Several striking features of the distributions are evident on the

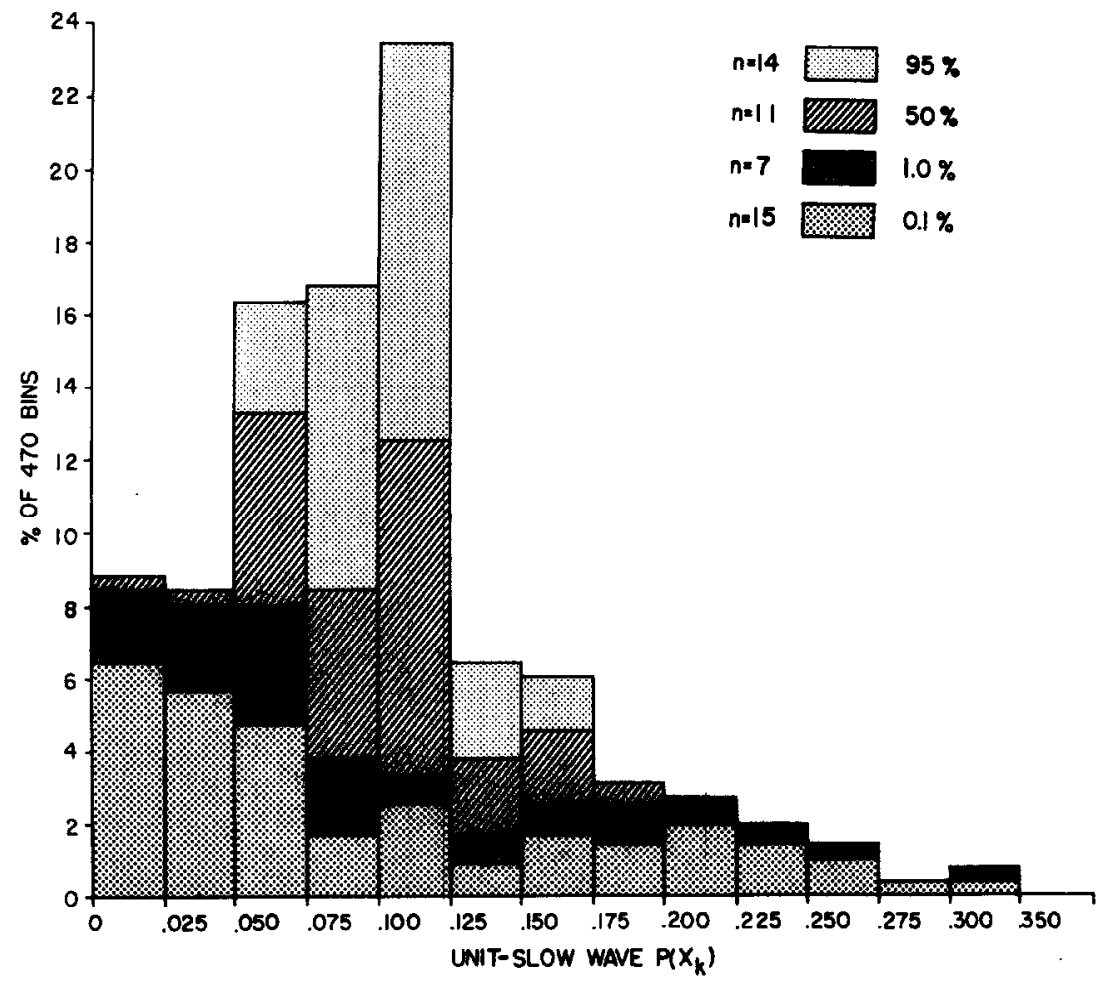

Fig. 2. Summary graph of phasegram bin probabilities. Neuronal data compared to the random model. Data taken from 47 phasegrams, each 10 bins wide and representing a different hippocampal neuron ( $n-$ number cells). Percentage figures (upper right) indicate significance levels for rejection of the null hypothesis. Patterns of only 1.0 and $0.1 \%$ phasegrams were considered nonrandom. See text. 


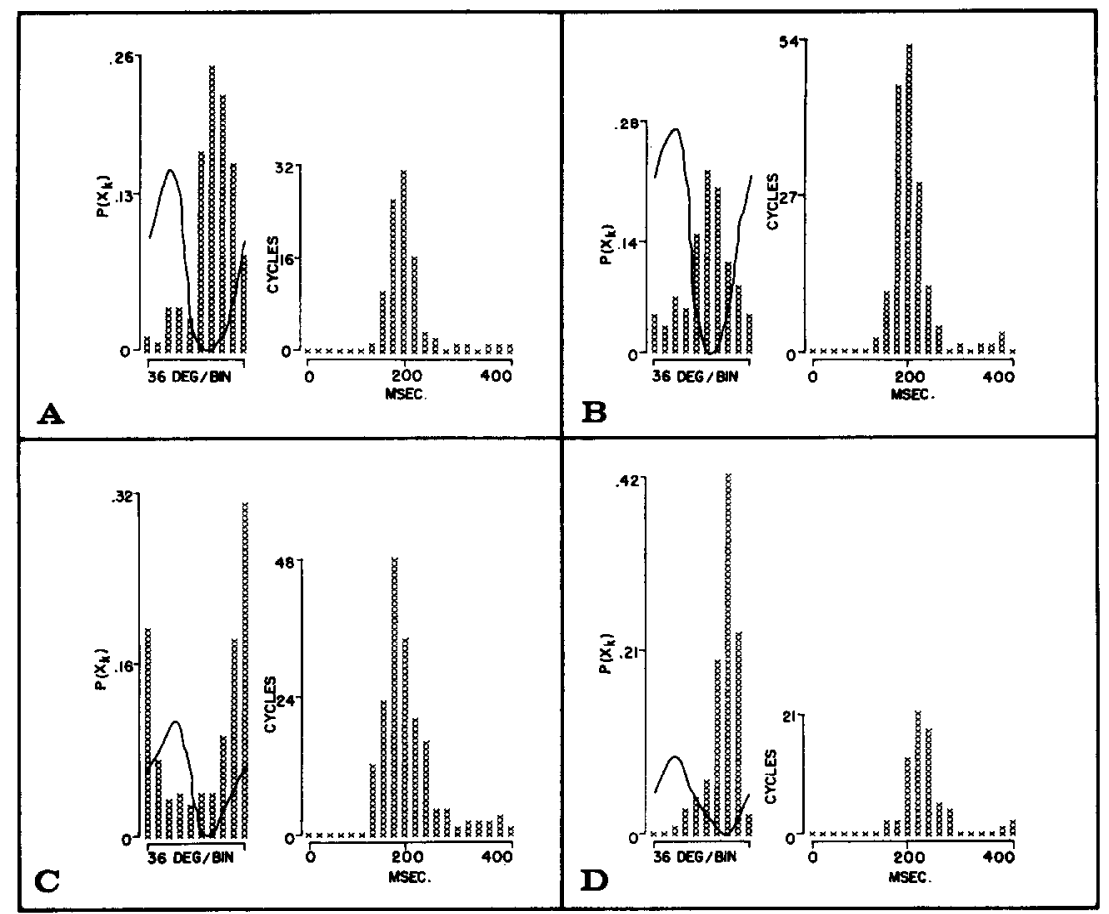

FIg. 3. Phasegrams and cycle histograms for spikes and local slow waves sampled from four different cells along a $300-\mu \mathrm{m}$ track in CA1 stratum oriens extending into stratum pyramidale. Patterns are nonrandom $(P<0.01)$. Note time variations in local slow waves. See text for discussion of spike-slow wave phase relations.

graph. First, the peak value indicates the most common bin probability value was 0.100 , the value expected for nonbiased bins in phasegrams consisting of 10 bins. The value characterized phasegrams of low confidence level categories: phasegrams $(95 \%, 50 \%)$ that were not different from the random model. Second, the range of bin probability values was widest for phasegrams in high confidence level groups $(1.0 \%, 0.1 \%)$ which is expected. Nonrandom phase patterns were characterized by numerous biased bins whereas random patterns wcrc not. Bins biased for spike occurrence $\left(P\left(X_{K}\right)>0.100\right)$ or nonoccurrence $\left(P\left(X_{K}\right)<0.100\right)$ were prominent in phasegrams of nonrandom data.

Table 1 lists properties of firing patterns grouped according to confidence levels. Average firing frequencies, numbers of spikes per slow wave cycle, and other features were quite similar among the groups. Measures listed in the table were not adequate to differentiate patterns classified according to confidence levels.

Anatomical Correlates. The site of unit recording within the cornu Ammonis was important. Nonrandom patterns were recorded from 22 of 
TABLE 1

Spike, Phasegram, and Cycle Histogram Data

\begin{tabular}{|c|c|c|c|c|}
\hline Phasegram class & $P<0.001$ & $P<0.01$ & $P<0.50$ & $P<0.95$ \\
\hline No. phasegrams & 18 & 4 & 9 & 12 \\
\hline Average no. spikes/sec & 9.7 & 11.4 & 14.1 & 9.4 \\
\hline $\begin{array}{l}\text { Average no. spikes/slow } \\
\text { wave cycle }\end{array}$ & 2.1 & 2.2 & 2.8 & 1.9 \\
\hline $\begin{array}{l}\text { Average of cycle } \\
\text { histogram peak times } \\
\text { (msec) }\end{array}$ & 208 & 200 & 198 & 206 \\
\hline $\begin{array}{l}\text { Average no. slow waves } \\
\text { in sample }\end{array}$ & 157.1 & 108.0 & 167.3 & 160.8 \\
\hline $\begin{array}{l}\text { Average no. spikes in } \\
\text { sample }\end{array}$ & 234.7 & 198.5 & 274.1 & 275.5 \\
\hline $\begin{array}{l}\text { No. narrow band } \\
\text { phasegrams ( } \leq 5 \text { bins } \\
\text { exceed half-amplitude } \\
\text { value of peak bin) }\end{array}$ & 18 & 2 & 0 & 0 \\
\hline $\begin{array}{l}\text { No. wide band } \\
\text { phasegrams ( }>5 \text { bins } \\
\text { exceed half-amplitude }\end{array}$ & & & & \\
\hline value of peak bin) & 0 & 2 & 9 & 12 \\
\hline No. recordings from $\mathrm{CAl}$ & 18 & 3 & 8 & 4 \\
\hline $\begin{array}{l}\text { No. recordings from } \\
\text { subicular region }\end{array}$ & 0 & 0 & 1 & 4 \\
\hline $\begin{array}{l}\text { No recordings from } \\
\text { area dentata }\end{array}$ & 0 & 1 & 0 & 4 \\
\hline
\end{tabular}

47 cells located in the dorsal CA1 or the area dentata. The hemisphere within which reference slow waves were sampled was immaterial. Nonrandom phase patterns were obtained from spikes recorded in one hemisphere and slow waves sampled elsewhere in the same or opposite hemisphere (Fig. 5). Because the midbrain transection was performed before eserine administration, ascending fibers from structures caudal to the midbrain were not necessary for induction, maintenance of the bilateral regular waves, or unit firing patterns.

Nonrandom phase patterns were generated by presumably closely spaced cells (Fig. 1C). In five different electrode penetrations, nonrandom phase patterns were obtained from different cells located along a short electrode track ( $400 \mu \mathrm{m}$ or less). Data from four cells recorded along a short track extending from stratum oriens into stratum pyramidale of CA1 are illustrated in Fig. 3. The nonrandom distributions appear to differ from each other. Because the local slow wave phase is a function of recording tip location $(33,34)$, absolute spike-slow wave phase value comparisons are not meaningful unless slow wave and spike signals are stationary in 


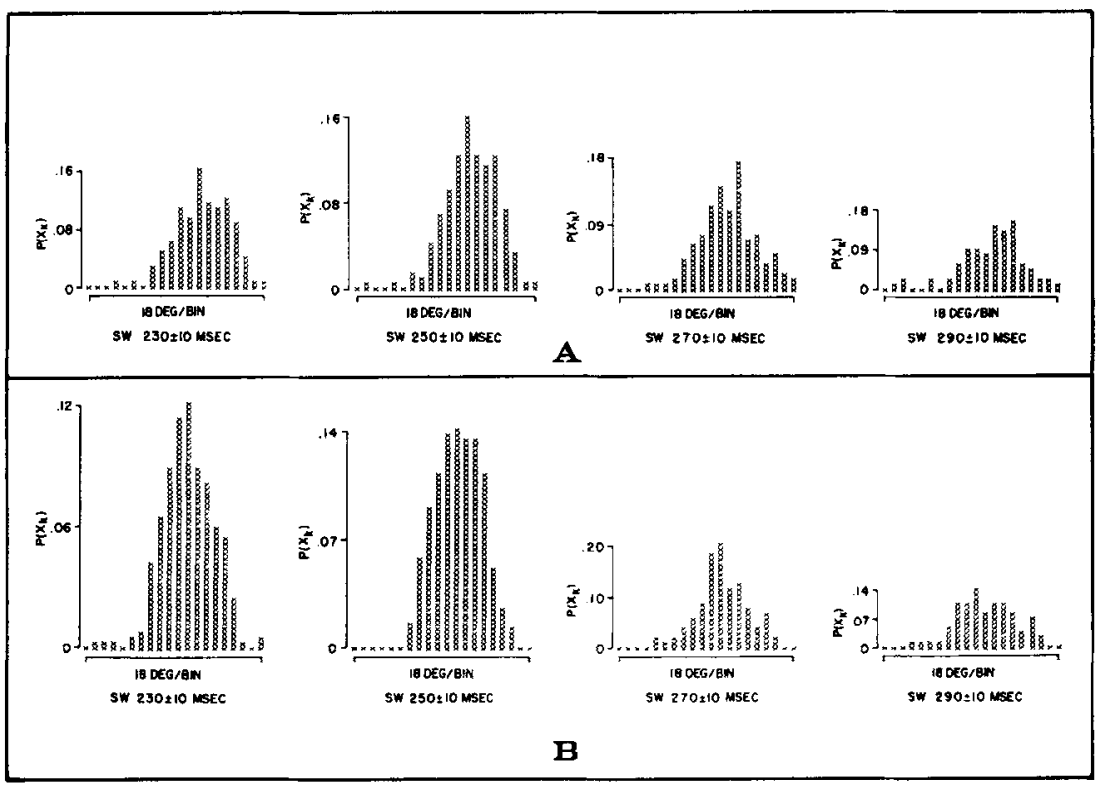

FIc. 4. Filtered data phasegrams for CA1 cell. Spike-slow wave phase measurements only made for local slow waves (SW) with stated durations. Distributions within A or B do not differ significantly $(P>0.05)$. Data of $B$ sampled 9 min $4 \mathrm{sec}$ after A. Left two distributions of A differ significantly $(P<0.001)$ from corresponding ones of $B$ whereas right two distributions do not.

time. The seemingly different phase values for the cells may be similar if the spikes were measured against the same reference signal.

Phasegram Decompositions. The possibility existed that the patterns were a function of slow wave duration. As can be deduced from the cycle histograms (Figs. 1 and 3 ) the slow waves are not constant in duration, and in the strictest sense so-called regular waves have considerable jitter. Following digital filtering of slow waves, separate phasegrams were assembled for spikes concurrent with waves of different durations. Sixtyeight second samples of nonrandom spike-slow wave data (10 cells) were processed. In no case did the nonrandom decomposed phase patterns for a train differ (all $P>0.05$ ) even though slow wave periods varied by as much as 100 msec. No pattern was ever observed to change from nonrandom to random or vice versa as a function of slow wave duration. However, changes within nonrandom patterns (Fig. 4) were observed as considered below.

A comparison of 136 second samples taken from the same train but with a lag of nearly nine minutes between the first and second sample indicates certain phasegram differences. The left two distributions of Fig. $4 \mathrm{~A}$ compared to corresponding ones of Fig. 4B differ significantly $(P<$ 
$0.001)$. Distributions within Fig. $4 \mathrm{~A}$ or $\mathrm{B}$ do not differ $(P>0.05)$. A similar result was obtained for cross comparisons of the right two distributions. These findings indicate mixed phase patterns (some similar, some not) for slow waves of similar duration but at different sample times.

Interspike Interval Shuffing to Break Sequential and Parallel Time Relations between Spikes and Slow Waves. Consistent nonrandom phase patterns indicate that the spikes occurred more frequently as the slow wave duration decreased. If the sequential order of spikes is broken by matching spikes with nonconcurrent slow waves, then the phase patterns should be significantly different.

Shuffled data phasegrams were constructed according to the following plan. A random-number generator was used to match sampled spikes with slow waves. First, $V_{r}$, the duration of a single slow wave, was randomly selected from a list of slow wave values. Another random number was used to elect $V_{b}$, the time value from the start of a slow wave to the first spike of a cycle, from a list. Because $V_{i}$ and $V_{r}$ were selected randomly, the two would overlap in real time only by chance $(1 \div$ the number

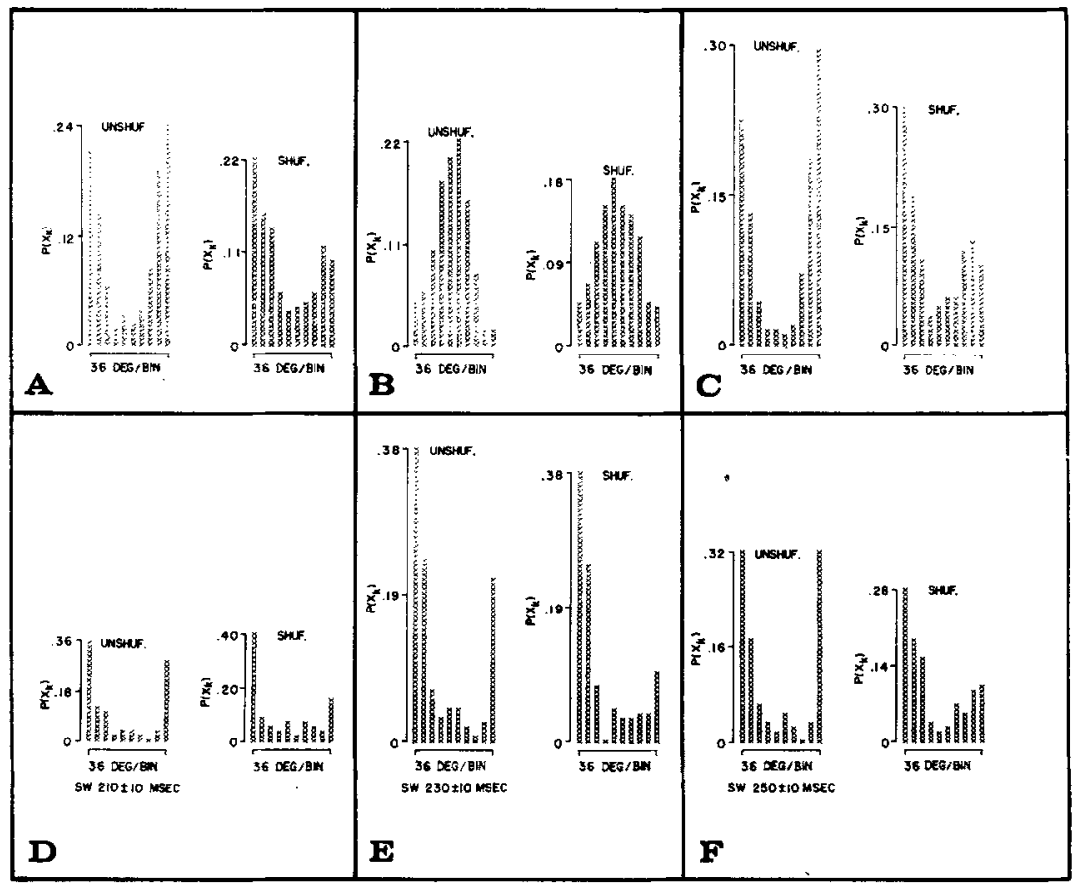

Fig. 5. Phasegrams of unshuffled and shuffled data (A-F) from four different CAI cells in stratum pyramidale. Filtered data from one cell, D-F. Local slow waves for A, C, D-F. Ipsilateral slow-wave electrode in fascia dentata for B. Shuffled and unshuffled data patterns differ significantly $(P<0.01)$. Sample period, $68 \mathrm{sec}$. 
of slow waves in the sample). Other random numbers were used to select list positions giving the number of spikes per slow wave cycle and remaining interspike interval times. Each sampled datum value was used only once, but all spike and slow wave data were selected. Shuffled and unshuffled data distributions differed in sizes as a result of cases where summed interspike intervals exceeded the cycle period and hence were discarded. The shuffled data phasegrams match slow waves with nonconcurrent spikes, thus destroying any sequential or parallel time relations that might have existed between concurrent spikes and slow waves.

Shuffed and unshuffled data phasegrams were compared using the $\chi^{2}$ test. Shuffling nonrandom data resulted in phasegrams having lower peaks and shallower troughs (Fig. 5). Significant differences $(P<0.05)$ between shuffled and unshuffled data phasegrams occurred for all 11 tested nonrandom patterns. Shuffling data of random patterns were immaterial, as could be expected. Results of this test complement the filtered data findings and provide evidence that CAl spikes were both phase modulated and not the result of coincidence.

\section{DISCUSSION}

The cells and mechanisms generating rhythmical slow waves are unknown. Rhythmical slow wave generators $(4,17,34)$ and nonrandomly firing cells are located in CA1 of the rabbit hippocampus. Firings phasemodulated to slow waves, as demonstrated in this study, indicate both are related by at least one common timing mechanism. Because firings and the waves are generated in the same hippocampal region, the timing mechanism may be operational in the same cell. Intracellular recordings of hippocampal pyramidal cells (13) show regular waves. Possibly, these neurons generate the slow waves, but other alternatives exist.

The spike-slow wave phase patterns could also indicate correlated signals of different cell types, phase-related responses of locally interacting neurons, or activities correlted by chance or coincidence. Coincidence can be excluded because similar phase patterns at different slow wave durations as observed would be most unlikely. Correlated signals of different cell types cannot be ruled out because the septum projects fibers to a variety of hippocampal structures and a medial septal (24) or thalamic (9) lesion abolishes the waves. This finding seems to exclude the clocking element being derived solely from hippocampal neurons but does not indicate which cells generate the phase-correlated potentials.

The histology and findings of nonrandomly firing cells along a short electrode track seem to rule out exclusive recordings from basket cells which are thought to be inhibitory to pyramidal cells $(1,2)$. The nonrandom patterns exist only because of firings and nonfirings, the latter 
being as essential as the former. Possibly the firings were from superficial pyramidal cells (23), most numerous in animals showing a prominent theta rhythm. The cells appear to be arranged in functional clusters and may be similar to theta cells of the rat (12).

Cluster size and shape may be defined by the field size and distribution pattern of basket cell terminations. Pyramidal cells receiving numerous basket cell terminals would be under more influential inhibitory control $(1,2)$ and have sharply defined phase relations not only to the slow waves but, more importantly, to the firings of each other (Fig. 1C). Those at a cluster's edge receiving fewer terminals or an admixture from different basket cells would have weaker or random phase relations. Hippocampal feedforward inhibition (2) and recurrent inhibition (28) may shape nonfiring periods whereas rebound excitation (21) would drive units to fire.

How contributions from the septum (24), subicular and entorhinal structures (8), and other brain regions (9) are precisely involved in the specific cellular actions awaits further definition and localization of the slow wave generator mechanism. Certainly the nonrandom spike-slow wave phase relations dependent on firing and nonfiring periods were not contingent on caudal brain stem ascending noradrenergic inhibitory (27) and excitatory fiber systems in animals receiving eserine.

\section{REFERENCES}

1. Andersen, P., J. C. Eccles, and Y. L $\phi$ Yning. 1964. Pathway of postsynaptic inhibition in the hippocampus. $J$. Neurophysiol. $27: 608-619$.

2. Andersen, P., G. N. Gross, T. Lomo, and O. Sveen. 1969. Participation of inhibitory and excitatory interneurons in the control of hippocampal cortical output, pp. 415-465. In "The Interneuron." M. A. B. Brazier [Ed.]. University of California Press, Los Angeles.

3. Batson, H. C. 1956. "An Introduction to Statistics in the Medical Sciences," pp. 38-42. Burgess, Minneapolis, Minnesota.

4. Bland, B. H., P. ANDERSEN, and T. Ganes. 1975. Two generators of hippocampal theta activity in rabbits. Brain Res. $94: 199-218$.

5. Bureš, J., M. Petrán, and J. Zachar. 1967. "Electrophysiological Methods in Biological Research," p. 704. Academic Press, New York.

6. Coyle, P., and L. Bowen. 1974. Neurophysiologic events monitored via computer asynchronous interface sense lines. J. Electrophysiol. Tech. 3: 48-51.

7. CoYle, P. 1976. Information in regular slow wave-unit potential phase measurements. In preparation.

8. Chronister, R. B., S. F. Zornetzer, J. J. Bernstein, and L. E. White, Jr. 1974. Hippocampal theta rhythm: interhippocampal formation contributions. Brain Res. 65 : 13-28.

9. Eidelberg, E., J. C. White, and M. A. B. Brazier. 1959. The hippocampal arousal pattern in rabbits. Exp. Neurol. $1: 483-490$.

10. Euler, C. von, and J. D. Green. 1960. Excitation, inhibition and rhythmical activity in hippocampal pyramidal cells in rabbit. Acta Physiol. Scand. 48: 110-125. 
11. Dumermuth, G. 1973. Numerical spectral analysis of the electroencephalogram, pp. 5A-33-60. In "Handbook of Electroencephalography and Clinical Neurophysiology." A. Rémond [Ed.]. Elsevier Scientific, Amsterdam.

12. Fox, S. F., and J. B. RANck. 1975. Localization and anatomical identification of theta and complex spike cells in dorsal hippocampal formation of rats. Exp. Neurol. 49 : 299-313.

13. Fujita, Y., and T. Sato. 1964. Intracellular records from hippocampal cells in rabbit during theta rhythm activity. $J$. Neurophysiol. $27: 1011-1025$.

14. Green, J. D. 1958. A simple microelectrode for recording from the central nervous system. Nature (London) 182: 962.

15. Green, J. D., and A. A. Arnuini. 1954. Hippocampal electrical activity in arousal. J. Neurophysiol. $17: 533-557$.

16. Green, J. D., and X. Machne. 1955. Unit activity of rabbit hippocampus. Amer. J. Physiol. $181: 219-224$.

17. Green, J. D., D. S. Maxwell, W. J. Schindeler, and C. Stumpf. 1960. Rabibit EEG "theta" rhythm: Its anatomical source and relation to activity in single neurons. J. Neurophysiol. 23 : 403-420.

18. HaRper, R. M. 1971. Frequency changes in the hippocampal electrical activity during movement and tonic immobility. Physiol. Behav. $7: 55-58$.

19. HARPER, R. M. 1973. Relationship of neuronal activity to EEG waves during sleep and wakefulness, pp. 130-153. In "Brain Unit Activity During Behavior." M. I. Phillips [Ed.]. C. C Thomas, Springfield, Illinois.

20. Jung, R., and A. E. Kornmuller. 1938. Eine Methodik der Ableitung lokalisierter Potentialschwankungen aus subcorticalen Hirngebieten. Arch. Psychiat. Nervenkr. 109 : 1-30.

21. Kandel, E. R., and W. A. Spencer. 1961. Electrophysiology of hippocampal neurons. II. After-potentials and repetitive firing. J. Neurophysiol. 24 : 243-259.

22. Kawamura, H., and E. F. Domino. 1969. Differential actions of $m$ and $n$ cholinergic agonists on the brainstem activating system. Int. J. Neuropharmacol. 8: 105-115.

23. LORENTE DE Nó, R. 1934. Studies on the structure of the cerebral cortex. II. Continuation of the study of the ammonic system. J. Psychol. Neurol. 46: 113-177.

24. Mayer, C., and C. Stumpr. 1958. Die Physostigminwirking auf die HippocampusTätigkeit nach Septumlasionen. Arch. Exp. Pathol. Pharmakol. 234: 490-500.

25. Noda, H., S. Manohar, and W. R. Adey. 1969. Spontaneous activity of cat hippocampal neurons in sleep and wakefulness. Exp. Neurol. $24: 217-231$.

26. RaNck, J. B., JR. 1973. Studies on single neurons in dorsal hippocampal formation and septum in unrestrained rats. Part I. Behavioral correlates and firing repertoires. Exp. Neurol. $41: 461-531$.

27. SEgaL, M., and F. E. Bloom. 1976. The action of norephinephrine in the rat hippocampus. III. Hippocampal cellular responses to locus coeruleus stimulation in awake rat. Brain Res, $107: 499-511$.

28. Spencer, W. A., and E. R. Kandet. 1961. Hippocampal neuron responses to selective activation of recurrent collaterals of hippocampal axons. Exp. Neurol. 4: 149-161.

29. StumPr, C. 1965. Drug action on the electrical activity of the hippocampus. Int. Rev. Neurobiol. 8: 77-138.

30. VAnderwolf, C. H. 1975. Neocortical and hippocampal activation in relation to behavior. J. Comp. Physiol. Psychol. 88 : 300-323. 
31. Vanderwolf, C. H., R. Kramis, L. A. Gillespie, and B. H. Bland. Hippocampal rhythmic slow wave activity and neocortical low voltage fast activity: relations to behavior, pp. 101-128. In "The Hippocampus," Vol. 2. R. L. Isaacson and K. H. Pribram [Eds.]. Plenum, New York.

32. Winson, J. 1972. Interspecies differences in the occurrence of theta. Behav. Biol. 7: $479-487$.

33. Winson, J. 1974. Patterns of hippocampal theta rhythm in the freely moving rat. Electroencephalogr. Clin. Neurophysiol. 36: 291-301.

34. Winson, J. 1976. Hippocampal theta rhythm. II. Depth profiles in the freely moving rabbit. Brain Res. $103: 71-80$.

35. WISHAW, I. Q., and C. H. VANDERwolF. 1973. Hippocampal EEG and bchavior: Changes in amplitude and frequency of RSA (theta rhythm) associated with spontaneous and learned movement patterns in rats and cats. Behav. Biol. 8: 461-484. 